\title{
Acknowledgment to Reviewers of Tropical Medicine and Infectious Disease in 2021
}

\section{TropicalMed Editorial Office}

Citation: TropicalMed Editorial Office. Acknowledgment to

Reviewers of Tropical Medicine and Infectious Disease in 2021. Trop. Med Infect. Dis. 2022, 7, 17. https:// doi.org/10.3390/tropicalmed7020017

Published: 27 January 2022

Publisher's Note: MDPI stays neutral with regard to jurisdictional claims in published maps and institutional affiliations.

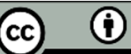

Copyright: $@ 2022$ by the author. Licensee MDPI, Basel, Switzerland. This article is an open access article distributed under the terms and conditions of the Creative Commons Attribution (CC BY) license (https://creativecommons.org/licenses/by/4.0/).

MDPI AG, St. Alban-Anlage 66, 4052 Basel, Switzerland

Rigorous peer-reviews are the basis of high-quality academic publishing. Thanks to the great efforts of our reviewers, TropicalMed was able to maintain its standards for the high quality of its published papers. Thanks to the contribution of our reviewers, in 2021, the median time to first decision was 17 days and the median time to publication was 42 days. The editors would like to extend their gratitude and recognition to the following reviewers for their precious time and dedication, regardless of whether the papers they reviewed were finally published:

Abhilasha Karkey

Abia Akebe Luther King

Adela Enache-Angoulvant

Adina Turcu-Stiolica

Ahmad Mourad

Ákos Jerzsele

Alberto Arnedo-Pena

Aldemir B. Oliveira-Filho

Aleksandra Burkowska-But

Alessandro Feola

Alice Michie

Amar Debbouche

Amina Khatun

Amy Gilbert

Ana Cecília Ribeiro Cruz

Ana Perisin

Anders C. Boyd

André P. Wolff

Andrea Cassoni

Andrea Cioffi

Andrea Coppola

Andrea Rossanese

Andreas Neumayr

Andrew Peters

Andrew Pike

Androula Pavli

Andrzej Tukiendorf

Andy Alhassan

Angela Barbosa

Anisuzzaman Anisuzzaman

Annetta Zintl
Annette Jurke

Anthony Guihur

Antonia Efstathiou

Antonio Baldassarre

Antonio Carlos Paes

Antonio Gregorio Dias Junior

Antonio Zurita

Apiporn Thinkhamrop Suwannatrai

Armanda Bastos

Assaf Rokney

Attila D. Sándor

Augustin Kadima Ebeja

Ayman Khattab

Ayman Mahmoud

Baicus Anda

Bayodé Roméo Adégbitè

Bela Kocsis

Benjamin Cull

Bethany Hedt-Gauthier

Bettina Wollanke

Bo Niklasson

Bongju Kim

Bronislava Vichova

Carla Bianca Luena Victorio

Carlo Genovese

Carmen de Kock

Carmen Solcan

Carol Yen Chin Lin

Carolina Davies

Cassiano Felippe Gonçalves-de-

Albuquerque

Catrin Moore 
Chee Keng Mok

Cheng-Chieh Yen

Christian Auer

Christian Nsanzabana

Claudia Marotta

Conxita Mestres

Daniela Boccolini

Danilo Oliveira Carvalho

David A. Edelman

Davide T. Mosca

Delia Mirela Tit

Denis Victorovich Tikhonenkov

Denise Harrison

Dimitris C. Chatzopoulos

Dinesh Subedi

Dolly J. Katz

Dominik Łagowski

Dominika Guzek

Donato Traversa

Dragana M. Stanojevic

Edoardo Pozio

Eiji Arakawa

Elisa García-Vázquez

Elisabetta Razzuoli

Elivelton Da Silva Fonseca

Elvina Viennet

Emilie Javelle

Emilie Vallée

Emmanouil Magiorkinis

Eric J. Nilles

Eskild Petersen

Eto Silas Fernandes

Ezgi Akdesir

F. Matthew Kuhlmann

Fabio Gomes

Federica Fogacci

Ferdinando Agresta

Francesca Baratta

Francesca Macchi

Francesco Castelli

Francesco Di Gennaro

Franco Mutinelli

Frank Dressler

Galya Ivanova Gancheva

George Panos

Germán Kopprio

Gianluca Piatelli

Gillian Gile

Giuseppe Miserocchi

Gouri Rani Banik

Grzegorz Woźniakowski
Guoju Hong

H.M. Manukumar

Han Naung Tun

Hayden Hedman

Hedwin Kitdorlang Dkhar

Heinz Mehlhorn

Hélcio Gil-Santana

Helene-Mari Van Der Westhuizen

Henrique Pereira

Heverton Dutra

Hiroshi Sato

Hiroyasu Sakai

Huibin Lv

Huijun Chih

Hyun-Ok Song

Ian Hodgson

Ian Mendenhall

Ibrahim E.A. Abbas

Inuk Jung

Ioannis A. Giantsis

Ioannis Giantsis

Iosif Marincu

Ismael Maatouk

Itziar Familiar

Ivana Grgić

Jacek Żmudzki

Jaeson Calla

Jaffar Abbas

Jaindra Nath Tripathi

James L.L. Occi

Jamilah Meghji

Jana Katuchova

Janani Iyer

Jean Perriot

Jean Vanderpas

Jean-Bernard Duchemin

Jef Van Den Ende

Jeffrey Bara

Jennifer D. Foulke-Abel

Jesús Navas

Jochen Hofstaetter

John L. Mokili

John McBride

John R. Rohde

Juan C. de la Torre

Julián Solís García Del Pozo

Julien Claude

Justyna Dunaj

Jyothi F. Nagajyothi

Kai J. Jonas

Karen E. Kempsell 
Karin Leder

Karolina Kot

Karoun Bagamian

Kasha Priya Singh

Katarzyna Bartosik

Kathryn Marie Jones

Kazuki Shimizu

Kempe Ronald Hope

Kerry Redican

Konstantinos Arsenopoulos

Kovács Nóra

Krishna Hort

Krishna Kishore Umapathi

Kristine Gebbie

Krzysztof Orczyk

Kuldeep Gupta

Kun-Hsien Tsai

Kurt G.M. De Cramer

Laura E. Via

Laurence Toutous Trellu

Lavinia Schuler Faccini

Leandro Ferracini Cabral

Leon Hugo

Leona Gilbert

Leonardo Pagani

Liam Reynolds

Lingpeng Shan

Lingxiao Xie

Lisiane Morelia Weide Acosta

Lizette Koekemoer

Lorna Leal

Luc Paris

Luca Busetto

Luca Coppeta

Luca Villa

Lucie Paloque

Luigi Meccariello

Luis Chaves

Magnus Grabe

Mahmoud Kandeel

Manuela Silva

Māra Pilmane

Marcello Tagliavia

Marcelo Andreetta Corral

Marcelo Palma Sircili

Marcos Bryan Heinemann

Marcus Scotti

Maria Alfonsa Cavalera

María J. García-Murria

Maria Teresa Armúa-Fernández

Maria Teresa Manfredi
Marialaura Corrente

Marie Varloud

Marino Vilović

Marios Papadakis

Marisol Ocampo

Marius Masiulis

Martin Taylor

Masaki Ota

Mateusz M. Plucinski

Mathieu Nacher

Matteo Bolcato

Matteo Riccò

Matthew Grant

Max Bachmann

Max Carlos Ramírez Soto

Melissa Agsalda-Garcia

Merete Markvart

Michael Padget

Michał Kazimierz Chojnicki

Michel Ledizet

Michele Fiore

Michele Lunardi

Miklós Bitay

Milan Kolář

Mohammad Abdul Motalib Momin

Mohammed Grawish

Mohammed Inayathullah

Monia Cocchi

Monika Pogány Simonová

Moosa Patel

Muhammed Afolabi

Mutsuyo Takayama-Ito

Nahed M. Abdel-Haq

Natalia Sumbatyan

Ngaio Richards

Nicole Gottdenker

Nikolaos A. Chrysanthakopoulos

Nobuko Tuno

Nobumichi Kobayashi

Norihiro Nishida

Nurudeen Olalekan Oloso

Olga Pawełczyk

Olga Perovic

Oliver Denis

Pandji Wibawa Dhewantara

Paolo Parente

Parisa Gazerani

Patricia Silvia Romano

Pau Bosch-Nicolau

Paul S. Weiss

Paulo Bettencourt 
Pawin Padungtod

Peter Zarb

Petr Horák

Phil LoVerde

Pier Luigi Acutis

Piera Mazzone

Pilar Foronda

Pin-Kuang Lai

Primrose Beryl Gladstone

Rachael Aubert

Raffaele Vitiello

Ravesh Singh

Regev Cohen

Regina Rowe

Remigiusz Gałęcki

Rhea Longley

Richard Trevor Wilson

Roberto Calisti

Robyn Stoddard

Rodolfo Capanna

Rodrigo Almeida-Paes

Roger Nasci

Roger Teck

Rogério Rodrigues

Ronaldo Cesar Borges Gryschek

Rose Njemini

Ryan D. Cooper

Saito Nobuo

Salvatore Giovanni De Simone

Sandeep Kumar

Satu Hepojoki

Saurabh RamBihariLal Shrivastava

Sebastian Kirchner

Shailesh Kumar

Sheikhpour Mojgan

Shylo R. Johnson

Silmara Marques Allegretti

Silvia Mascolo

Silvia S. Chiang

Sonu Subudhi

Sophie Huddart

Stefano Catalano

Stefano Veraldi
Steffen Flessa

Stella Maris González Cappa

Stephen Graham

Stephen Muhi

Steve Carlan

Steven Stoddard

Sudhanshu Abhishek

Sulaiman Lakoh

Suman Majumdar

Susan Beth Rifkin

Taichiro Takemura

Takashi Yoshiyama

Terry A. Klein

Theresa Coetzer

Thiru Vanniasinkam

Thomas Robert Shelite

Thomas Zoller

Timothy Erickson

Timothy P. Algeo

Ting Li

Tomas Jelinek

Tomasz Blicharski

Tomislav Meštrović

Tomohiro Ishikawa

Tony Le Gall

Tony Schountz

Toshio Hattori

Veronika Katharina Jaeger

Victor Arendt

Villa Simone

Vincent Delorme

Vishnu Revuri

Vitrai József

Vladimir Savic

Whitney Qualls

Wilman Carrillo

Xavier Vallès

Xinzhuan Su

Xupeng Hong

Yalemzewod Gelaw

Yang Hong

Yueh-Juen Hwu

Zsuzsanna Füzesi 